\title{
Feasibility of laparoscopic surgery in the management of rare intrahepatic perforation of the gall bladder and liver abscess
}

\author{
Vishwas Johri, Vimal Kumar Dhaduk, Nikunj Jain, Prasanna Kumar Reddy \\ Apollo hospital, Chennai, India.
}

Keywords: Gall bladder perforation; intrahepatic gall bladder perforation; liver abscess

\section{Introduction}

Gall bladder perforation is an uncommon complication of acute cholecystitis in present time due to increased number of cholecystectomies being offered at an early disease stage and laparoscopic cholecystectomy being a straight forward and established gold standard procedure. Its association with cholecystohepatic communication and liver abscess is a rare complication. Opinions are divided amongst various authors regarding the classification and management of this entity. We report our experience in the management of this complication using minimally invasive surgical techniques and our opinion regarding classification and its optimal management.

\section{Case presentation 1}

A 45 year old gentleman presented with intermittent episodes of upper abdominal pain over 4 years which increased in severity in the last 2 weeks. He had fever for 8 days. It was also associated with loss of appetite. There was no history of jaundice. On examination the patient was afebrile. His abdomen was soft with mild tenderness in epigastrium. A CECT abdomen showed features of chronic cholelithiasis with intra hepatic extension of gall bladder with breech in posterior wall [Fig.1a] and abscess formation in segment 5 and 6 of liver[Fig. 1b]. The patient was offered laparoscopic cholecystectomy with drainage of liver abscess. Intra operatively a severely diseased thick walled gall bladder with frozen Calot's triangle was found. There was an impacted stone at the neck of gall bladder. The patient withstood the procedure well. He was discharged on post operative day 1 and his abdominal drain was removed on day 3 as an outpatient.

\section{Case presentation 2}

A 55 year old gentleman was diagnosed with cholelithiasis and choledocholithiasis on routine work up for jaundice. He

\footnotetext{
Correspondence: Vishwas Johri

E-mail: vishwas@gmail.com

Received: 24-07-2019 Accepted: 29-10-2019

iD https://orcid.org/0000-0002-3342-5449 DOI: http://doi.org/10.4038/sljs.v37i3.8566
}

underwent therapeutic ERCP and CBD stenting and was advised to report back for definitive surgery after 8 weeks. Six weeks later he presented to the emergency department with abdominal pain fever with chills. On examination the patient had a temperature of $100.4^{\circ} \mathrm{F}$. He had an otherwise soft abdomen with tenderness in the right hypochondrium. A provisional diagnosis of cholangitis was made. Blood investigations showed haemoglobin- $8.5 \mathrm{gm} \%$, TLC- $8680 / 3$ $\mathrm{mm}$, albumin- $2.6 \mathrm{~g} / \mathrm{dl}$, ALP- $141 \mathrm{u} / 1$, calcium- $6.9 \mathrm{mg} / \mathrm{dl}$. The rest of the investigations were unremarkable. The US abdomen showed chronic cholecystitis and an abscess in right lobe of the liver. The CECT abdomen showed calculous cholecystitis with gall bladder fundus attached to the under surface of liver and colon and an abscess in segment 5 of the liver [Fig.2a]. The patient was optimised and a laparoscopic cholecystectomy and drainage of the liver abscess was done. Intra operatively a thick walled, gall bladder empyema with gangrenous changes was found. It was adherent to the duodenum and transverse colon. A large stone was found impacted at the neck of gall bladder [Fig. 2b]. Calot's triangle was severely inflamed and a perforation was found at the fundus which communicated with the liver abscess. The patient recovered well and the abdominal drain was removed on day 6 . He was discharged in good condition on day 12 .

\section{Discussion}

Gall bladder perforation is an infrequent but serious complication of cholecystitis. Its prevalence has decreased over time from $2-15 \%$ in earlier case reports to $0.8 \%$ in recent literature [1]. It may be attributed to increased availability and simplicity of laparoscopic cholecystectomy and also due to availability of better antimicrobial and analgesic agents.

It may develop early in the course of acute or in chronic cholecystitis or it may occur as late as several weeks after onset. Predisposing factors for gall bladder perforation include cholelithiasis, infection, malignancy, trauma, corticosteroid therapy, diabetes mellitus, impaired vascular supply, old age and male sex [1].

Development of a gall bladder perforation involves a series of events starting from obstruction of the cystic duct by a calculus. It leads to biliary stasis and gall bladder distension. Such persistently raised intraluminal pressure leads to 


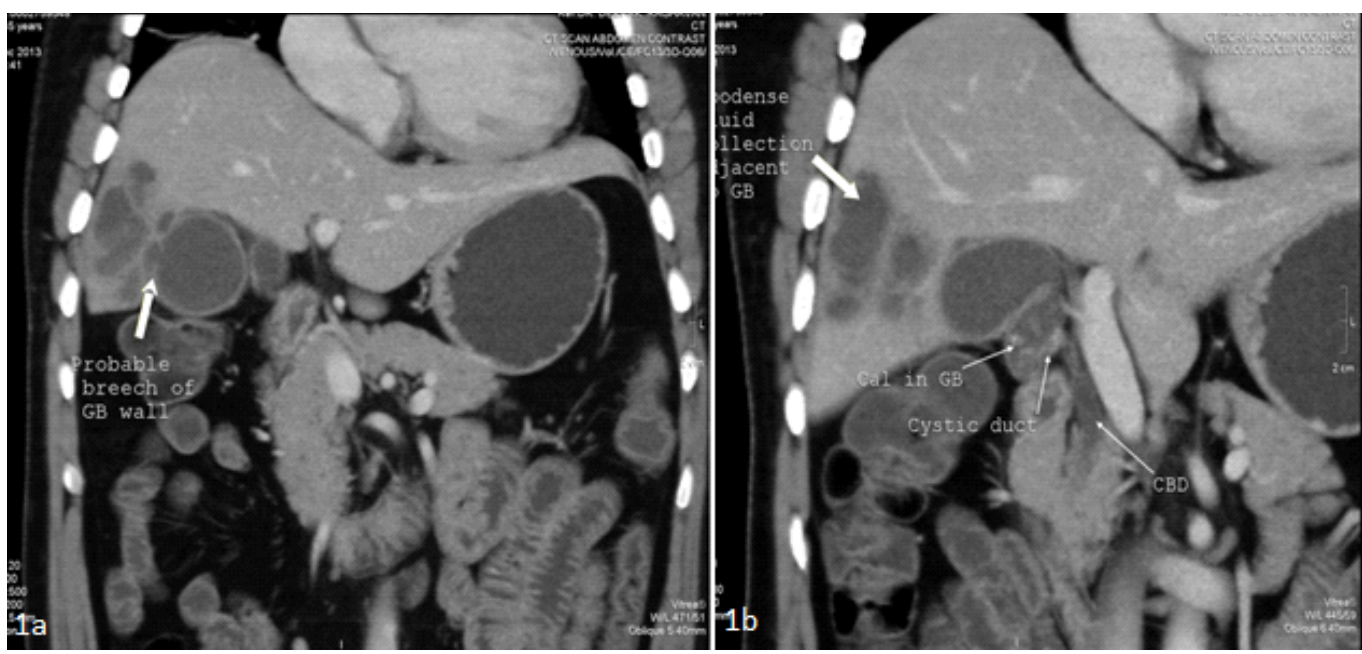

Figure 1a. CT scan showing breech in the posterior wall of the gall bladder.

Figure 1b. Intrahepatic gall bladder with changes of chronic cholelithiasis and abscess in segment 5 and 6 of liver.
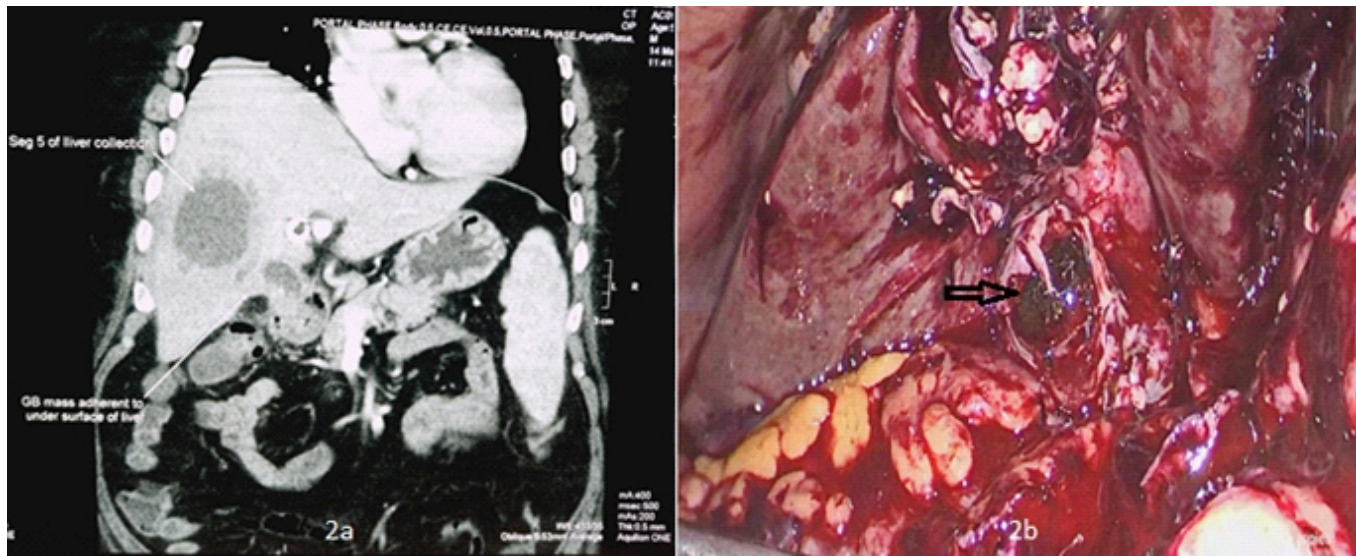

Figure 2a. CT scan showing intrahepatic gall bladder with abscess in segment 5 of liver.

Figure 2b. Intra operative image showing thick walled gall bladder with gangrenous changes in the fundal region and impacted stone in the neck of gall bladder (black arrow).

decreased venous and lymphatic drainage leading to eventual gall bladder wall necrosis and finally, a perforation. The most common site of a gall bladder perforation is the fundus because it is the most distal part of the gall bladder and has the lowest blood supply [2].

In 1952, Fletcher and Ravdin, while describing 44 cases of gall bladder perforation using Niemeier's method of classification, divided them as follows: type 1, acute free perforation into the peritoneal cavity; type 2, sub acute perforation with pericholecystic abscess; and type 3 , chronic perforation with cholecystoenteric fistula [1].

There are no classical symptoms and signs associated with gall bladder perforations. Patients may present acutely or may have an insidious onset. Right upper quadrant pain, fever, palpable right upper quadrant mass and tenderness may herald an acute onset. In contrast, patients may present with weakness, malaise, anorexia and a palpable right upper quadrant mass, mimicking a malignant lesion. Elevated liver enzymes, especially alkaline phosphatase levels are commonly documented. A sudden decrease in pain intensity may occur at the time of perforation due to decompression of the high intra-cholecystic pressure. As the majority of these features are also present in acute cholecystitis, it is difficult to differentiate clinically between patients with perforated gall bladders and those with uncomplicated acute cholecystitis [1]. Ultrasonography may be suggestive, but most findings are not specific for perforation.

These could be distension of the gall bladder and increased wall thickness or oedema. Perforation can be demonstrated unequivocally only by a CT or MRI scan. These investigations are also useful in locating other intraabdominal lesions. Since amoebic liver abscess is commonly encountered in India and other developing countries, these cases alert us to the possibility of the gallbladder being the primarily diseased organ, requiring cholecystectomy in addition to management of the abscess [3].

The treatment modality has not been fully established for gall bladder perforation with cholecystohepatic communication 
leading to liver abscesses. Most of the reported case reports from India have resorted to open cholecystectomy and drainage of the abscess [2]. Kochar et al have also advocated using open cholecystectomy in the first instance because of dense adhesions and poor anatomical detailing [1]. They have also advocated the use of conservative management with percutaneous drainage followed by cholecystectomy at a later date [1].

\section{Conclusion}

Intrahepatic perforation of gall bladder leading to liver abscess is a rare complication with around 20- 25 such cases reported in world literature so far. If the operating team is well versed in dealing with difficult cholecystectomies, laparoscopic cholecystectomy is a feasible first line option. If successfully done it brings about cure in a single sitting which helps to cut down cost, hospital stay and morbidity to the patient. It also offers all other benefits that are inherent to minimal access surgery like cosmesis and less pain. Staged procedures should be offered only to those patients who are poor candidates for surgery.
All authors disclose no conflict of interest. The study was conducted in accordance with the ethical standards of the relevant institutional or national ethics committee and the Helsinki Declaration of 1975, as revised in 2000 .

\section{References}

1. Kochar K, Vallance K, Mathew G, Jadhav V (2008) Intrahepatic Perforation of the gall bladder presenting as liver abscess: case report, review of literature and Niemeier's classification. Eur J Gastroenterol Hepatol 20: 240-244.

https://doi.org/10.1097/MEG.0b013e3282eeb520

2. T. Hussain, M. Adams, M. Ahmed, N. Arshad, M. Solkar. Intrahepatic perforation of the gallbladder causing liver abscesses: case studies and literature review of a rare complication. Ann R Coll Surg Engl 2016; 98: e88-e91. https://doi.org/10.1308/rcsann.2016.0115.

3 Nitin Agarwal, Pradeep Saini , Arun Gupta , Navneet Kaur, Suruchi Shresttha, Mohammad Shazib Faridi. Chronic hepatic abscess due to gallbladder perforation: three cases and exact nomenclature. Tropical Gastroenterology 2013; 34(3):199-202. http://dx.doi.org/10.7869/tg.135

\section{Learning Points:}

- A comprehensive preoperative workup including a good history, clinical examination laboratory and radiological investigations are quintessential in making a correct diagnosis of such rare presentations.

- Laparoscopic cholecystectomy and drainage of liver abscess is a viable and robust modality in the management in experienced hands.

- Staged procedures should be offered only to those patients who are poor candidates for surgery. 